This is the submitted version of the article:

Schaefer N., Garcia-Cortadella R., Martínez-Aguilar J.,

Schwesig G., Illa X., Moya Lara A., Santiago S., Hébert C., Guirado G., Villa R., Sirota A., Guimerà-Brunet A., Garrido J.A.. Multiplexed neural sensor array of graphene solution-gated field-effect transistors. 2D Materials, (2020). 7. 025046: - . 10.1088/2053-1583/ab7976.

Available at: https://dx.doi.org/10.1088/2053-1583/ab7976 


\title{
Multiplexed Neural Sensor Array of Graphene Solution-Gated Field-Effect Transistors
}

\author{
Nathan Schaefer ${ }^{1,2}$, Ramon Garcia-Cortadella, ${ }^{1,2}$, Javier Martínez-Aguilar ${ }^{3,4}$, Gerrit Schwesig ${ }^{5}$, Xavi Illa ${ }^{3,4}$, Ana \\ Moya Lara ${ }^{3,4}$, Sara Santiago ${ }^{6}$, Clement Hébert ${ }^{7}$, Gonzalo Guirado ${ }^{6}$, Rosa Villa ${ }^{3,4}$, Anton Sirota ${ }^{5}$, Anton Guimerà- \\ Brunet $^{3,4}$ and Jose A. Garrido ${ }^{1,8}$
}

\begin{abstract}
${ }^{1}$ Catalan Institute of Nanoscience and Nanotechnology (ICN2), CSIC and the Barcelona Institute of Science and Technology, Campus $\mathrm{UAB}$, Bellaterra, Spain

${ }^{2}$ Departament d'Enginyeria Electrònica, Escola d'Enginyeria, Universitat Autònoma de Barcelona, Bellaterra, Spain

${ }^{3}$ Instituto de Microelectrónica de Barcelona IMB-CNM (CSIC), Esfera UAB, Bellaterra, Spain

${ }^{4}$ Centro de Investigación Biomédica en Red en Bioingeniería, Biomateriales y Nanomedicina (CIBER-BBN), Madrid, Spain

${ }^{5}$ Bernstein Center for Computational Neuroscience, Ludwig-Maximilians-University Munich, Planegg-Martinsried, Germany

${ }^{6}$ Departament de Química, Universitat Autònoma de Barcelona, Bellaterra, Barcelona, Spain

${ }^{7}$ Inserm and Université Grenoble Alpes, Saint Martin d'Hères, France

${ }^{8}$ ICREA, Barcelona, Spain

E-mail: joseantonio.garrido@icn2.cat
\end{abstract}

Received xxxxxx

Accepted for publication $\mathrm{xxxxxx}$

Published xxxxxx

\begin{abstract}
Electrocorticography $(\mathrm{ECoG})$ is a well-established technique to monitor electrophysiological activity from the surface of the brain and has proved crucial for the current generation of neural prostheses and brain-computer interfaces. However, existing ECoG technologies still fail to provide the resolution necessary to accurately map highly localized activity across large brain areas, due to the rapidly increasing size of connector footprint with sensor count. This work demonstrates the use of a flexible array of graphene solution-gated field-effect transistors (gSGFET), exploring the concept of multiplexed readout using an external switching matrix. This approach does not only allow for an increased sensor count, but due to the use of active sensing devices (i.e. transistors) over microelectrodes it makes additional buffer transistors redundant, which drastically eases the complexity of device fabrication on flexible substrates. The presented results pave the way for upscaling the gSGFET technology towards large-scale, high-density $\mu \mathrm{ECoG}$-arrays, eventually capable of resolving neural activity down to a single neuron level, while simultaneously mapping large brain regions.
\end{abstract}

Keywords: Multiplexed $\mu$ ECoGs, graphene solution-gated field-effect transistor, flexible probes, neurosensing

\section{Introduction}

Exploration of novel materials and improved micro- and nanofabrication techniques are bringing up a new class of braincomputer interfaces (BMIs) which promise to revolutionize neuroprosthetics and unveil the underlying vast functionalities of the brain. Impressive breakthroughs have been recently achieved in motor control rehabilitation, understanding the mechanisms for learning and formation of memory, treating neuropsychiatric disorders (e.g. depression) and synthesizing audible speech using machine learning algorithms, emphasizing the immense potential BMIs have.[1-10] While the presented capabilities to interface with the brain are already very impressive, they mostly rely on relatively simple epi-cortical electrode arrays with low number of recording sites and large inter-site spacing, and are unsuitable for long chronic implantation. Higher cognitive functions do typically arise from a complex interplay of activity in several brain regions at once, therefore a detailed analysis of the neural activity underlying such functions would require sensor arrays recording from all involved areas with high local resolution in each of them. Current commercially available neural sensor arrays fail to provide the high sensor counts necessary for such endeavor, mostly due to the technologic challenge of excessive wiring with increasing array size, which could only 
be overcome by the employment of multiplexed read-out circuitry. Multiplexing strategies are not new to the field of neuro-sensing and have previously been used as a versatile tool to manage large amounts of recording sites. Most of the emerged technologies are based on a CMOS-compatible monolithic integration of recording electrodes and read-out electronics to minimize connection distance for preserving signal integrity and maximizing the density of recording sites. Using rigid silicon substrates, however, restricts such concepts to applications in which device flexibility is expendable such as depth-probes or MEAs for ex-vivo recordings.[11-14] Yet, considering the extensive damage rigid depth-probes cause to the brain tissue, sets a clear limitation to the amount of insertable shanks and thus the simultaneously mappable brain regions. Here, electrocorticography offers a minimally invasive alternative with clear advantage on sensor resolution over large areas by using flexible $\mu \mathrm{ECoG}$ arrays to record from the surface of the cortex.[15] Still, so far only very few examples of multiplexed $\mu \mathrm{ECoG}$ arrays have been realized, due to the scarcity of durable materials for low-noise, highperformance switches compatible with flexible substrates as well as the high level of complexity such device fabrication requires. An example is the work of Rodgers which utilizes a combination of buffer and addressing transistor (ultrathin $\mathrm{Si}$ ) to process the signals of an array of 360 passive platinum electrodes.[16, 17] Using passive elements, i.e. electrodes, as sensors requires the implementation of an additional transducer (buffer transistor) to decouple the electrode from the read-out circuitry, which otherwise would lead to load currents affecting the electrodes performance. This additional complexity can be avoided by directly using active elements i.e. transistors to interface with the brain. Such approach has recently been explored for organic electrochemical transistors (OECTs) with the conductive polymer poly $(3,4-$ ethylenedioxythiophene):poly(styrenesulfonate)

(PEDOT:PSS) as channel material.[18, 19] However, it has only been used to selectively address different sites, but not yet to actually acquire signals in multiplexed operation, which might be due to the relatively low carrier mobility in PEDOT:PSS prohibiting the rapid switching needed for multiplexing.

As the brain consists of corrugated soft tissue moving at every heartbeat, highly flexible probes are imperative to create intimate interfaces for best signal quality and to avoid gradual cicatrizing of the neural tissue. In this work, we present flexible multiplexed $\mu$-ECoG arrays based on active sensing devices, namely graphene solution-gated field-effect transistors (gSGFETs). The gSFGET has emerged as one of the most promising technologies for brain-machine interfaces (BMIs) as it provides essential properties such as biocompatibility, chemical stability, mechanical flexibility and high signal-to-noise ratio.[20, 21] In particular, it has shown great potential for application in $\mu$-ECoG arrays with its high sensitivity over a broad frequency range $(0.001 \mathrm{~Hz}$ up to $10 \mathrm{kHz}$ ), making it an efficient transducer of both infra-slow and fast neural activity.[20, 22] While the gSGFETs suitability to provide high-quality recordings has already been proven for up to 16 recording sites in previous publications, its potential has never been evaluated for high-density arrays of large sensor count. Graphene is commonly considering as an ideal material for high-frequency application, as its reported mobilities up to $350000 \mathrm{~cm}^{2} \mathrm{~V}^{-1} \mathrm{~s}^{-1}$ for CVD grown graphene, can easily surpass materials such as $\mathrm{Si}$ and PEDOT:PSS with respective mobilities of $1400 \mathrm{~cm}^{2} \mathrm{~V}^{-1} \mathrm{~s}^{-1}$ and $0.01 \mathrm{~cm}^{2} \mathrm{~V}^{-1} \mathrm{~s}^{-1}$, which makes it an interesting candidate to consider for multiplexed devices.[23, 24] Moreover, we demonstrate that by using active sensing devices, the integration of multiplexing circuitry, i.e. buffer and switching transistors, is unnecessary, thus drastically easing the complexity of fabricating flexible multiplexed sensor arrays.

\section{Methods}

\subsection{Probe fabrication}

In a first step 10um thick biocompatible Polyimide (PI-2611 HD MicroSystems) was spun on 4' $\mathrm{Si} / \mathrm{SiO} 2$ support wafers and cured under nitrogen atmosphere at $350{ }^{\circ} \mathrm{C}$. The perpendicular metal lines of the array (columns/ rows) were patterned in two standard lift-off steps (negative photoresist AZ5214E, Clariant, Germany) with the metal deposited by ebeam evaporation (30nm Ti/300nm Au, $10 \mathrm{~nm} \mathrm{Ti} / 100 \mathrm{~nm} \mathrm{Au})$ and separated by a $2 \mu \mathrm{m}$ PI spacer layer. Interconnecting viaholes through the spacer layer were etched by oxygen plasma using a photolithographically defined protective aluminum mask (AZ5214E, 300nm Al). Then, single layer graphene, grown and transferred by Graphenea, is patterned by photolithography (H6512 photoresist) and etched by oxygenbased reactive ion etching (100W for $1 \mathrm{~min}$ ) to form the transistor channel area. A third metal layer $(20 \mathrm{~nm} \mathrm{Ni} / 200 \mathrm{~nm}$ $\mathrm{Au}$ ) is added to form sandwich contacts improving the gSGFET's durability and lowering its contact resistance due to work function matching. A subsequent thermal annealing step at $300^{\circ} \mathrm{C}$ in ultra-high vacuum has been found to reduce surface contamination from photoresist residues and improving the conformality of the Ni-graphene interface, thus improving contact resistance and device performance. To passivate the metal leads, a $2 \mu \mathrm{m}$ thick layer of SU8 epoxy photoresist (SU-8 2005 MicroChem) was deposited, leaving open windows in the channel regions to allow a direct electrolyte graphene interface. In a final step, the polyimide 
was structured by deep reactive ion etching using a photoresist etching mask (AZ9260, Clariant) and the flexible probes were mechanical peeled from the support wafer.

\subsection{Characterization and multiplexing setup}

Custom-build electronics were used for bias control and to convert the drain source current signal into voltage by a transimpedance amplifier (10k gain). The voltage signal was split into DC (frequency $<0.1 \mathrm{~Hz}$ ) and $\mathrm{AC}(0.1 \mathrm{~Hz}$ $<$ frequency $>5 \mathrm{kHz}$ ) components. The AC signal is amplified by an additional factor of 100 for the noise evaluation. The voltage read out was done by a standard data acquisition system (National Instruments DAQ-Card, USB-6363). For the multiplexed data acquisition, a similar system was used as for the probe characterization, however the gain of the AC stage was reduced by a factor of 10 to prevent saturation of the amplifier's dynamic range due to mismatch in transistor resistance. The digital output lines were used to address the ntype MOSFET switching matrix by applying either $+5 \mathrm{~V}$ for $\mathrm{ON}$ - and $\mathrm{OV}$ for OFF-state. All software to control the DAQCard and handle data acquisition is based on self-built python code.

\subsection{In-vivo experiments}

Long Evans rats (Charles River) were kept under standard conditions (room temperature $22 \pm 2{ }^{\circ} \mathrm{C}, 12: 12 \mathrm{~h}$ light-dark cycle, lights on at 10:00). Food and water were provided ad libitum. All experiments were performed in accordance with the European Union Directive 2010/63/EU as well as the German Law for Protection of Animals (TierSchG) and approved by the local authorities (ROB-55.2-2532.Vet_0216-170). Three adult rats ( 2 males, 1 female), 3-8 month of age, weighing in the range of $400-600 \mathrm{~g}$ were used in this study. In preparation of electrophysiological measurements, they were deeply anaesthetized with MMF (Midazolam $2 \mathrm{mg} / \mathrm{kg}$ ), Medetomidin $0.15 \mathrm{mg} / \mathrm{kg}$, Fentanyl $0.005 \mathrm{mg} / \mathrm{kg}$ ) and supplemented after $1 \mathrm{~h}$ with Isoflurane $0.5 \%-1 \%$ and Metamizol at $110 \mathrm{mg} / \mathrm{kg}$. After subcutaneous infiltration with Bupivacain the skin above the cranium was incised and the dorsal skull surface exposed. Craniotomies were performed bilaterally, with a maximum width of $5 \mathrm{~mm}$ and extending anterior-posteriorly between $+2 \mathrm{~mm}$ and $-8 \mathrm{~mm}$ with respect to bregma. The dura mater was opened and carefully resected. The craniotomies were subsequently covered with prepolymerized PDMS (Sylgard 184, Dow Corning) with mixing ratio 1:10 and fastened with Vetbond (3M). In one rat an additional $1 \times 1 \mathrm{~mm}$ craniotomy was performed over the cerebellum for the placement of a reference wire. For placement of the recording arrays the PDMS covers were flapped open partially and the gSGFET array was placed on the right hemisphere while the NeuroNexus array (E32-60010-100) was symmetrically positioned on the left hemisphere, (between ca -7 to $-3 \mathrm{~mm}$ from bregma) each partially covering the primary visual cortex. Subsequently the PDMS covers were flapped back to cover arrays and craniotomies. A reference wire $(\mathrm{Ag} / \mathrm{Ag}-\mathrm{Cl})$ was inserted either in the cerebellar craniotomy $(n=1)$ or temporal muscle $(n=2)$. Data from the NeuroNexus array was acquired at $25 \mathrm{kHz}$ using the eCube recording system (WhiteMatter LLC) while data from the gSGFET was acquired using custom build electronics described in the previous section. Anesthesia was kept at $0.5 \%$ isoflurane during the recordings of spontaneous activity and cortical spreading depression (CSD) and $2 \%$ isoflurane for the recording of optically evoked activity with reduced spontaneous activity. The CSD event was triggered by application of $1 \mathrm{uL} \mathrm{KCl}(3 \mathrm{mMol})$ with a glass-micropipette and Nanoject II injection device (Drummond Scientific) at ca $4 \mathrm{~mm}$ anterior to bregma, approximately $7 \mathrm{~mm}$ anterior to the closest site on the recording array. The optically evoked response was triggered by a contralaterally placed blue LED in front of the left eye, which delivered $100 \mathrm{msec}$ light pulses every 5 seconds.

\section{Results and discussion}

\subsection{Device performance and multiplexing methodology}

Scalable thin-film technology of $8 \times 8$ gSGFET sensor arrays was fabricated on 7um-thick flexible polyimide (PI) using 4inch support wafers. The layout of the probe can be seen in Figure 1a and consists of a stack of two metal layers constructing the perpendicular lines of the sensor grid with a separating PI layer in between. The 64 gSGFET sensors have single-layer graphene channels of $50 \mu \mathrm{m}$ x $50 \mu \mathrm{m}$ dimension and $400 \mu \mathrm{m}$ inter-site separation (detailed description of probe fabrication in experimental section). A picture of the final probe, after releasing it from the Si support wafer is shown in Figure 1c. In contrast to their solid-state counterparts, solution-gated transistors are being modulated through the creation of a charge double layer at the interface between transistor channel and an electrolyte solution. Potential fluctuations in the surroundings (e.g. neural activity) can modulate this double layer and result, in turn, in a shift of the Fermi level of the channel material, which is detectable as a current fluctuation. Graphene's ability to create a stable interface with aqueous solutions in combination with its high transconductance, resulting from its large interfacial capacitance and carrier mobility, makes the gSGFET an ideal device for bio-sensing.[20-22, 25-28] 
a

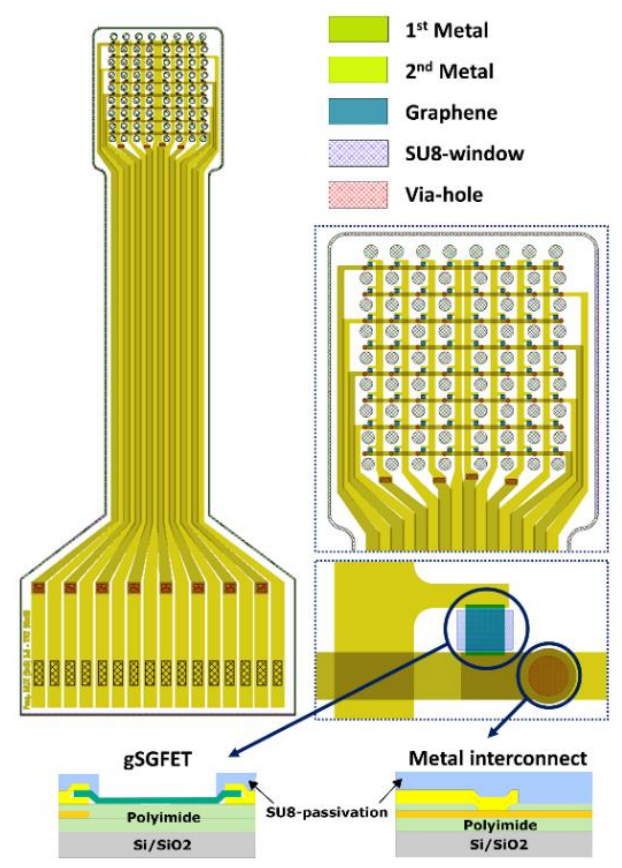

b
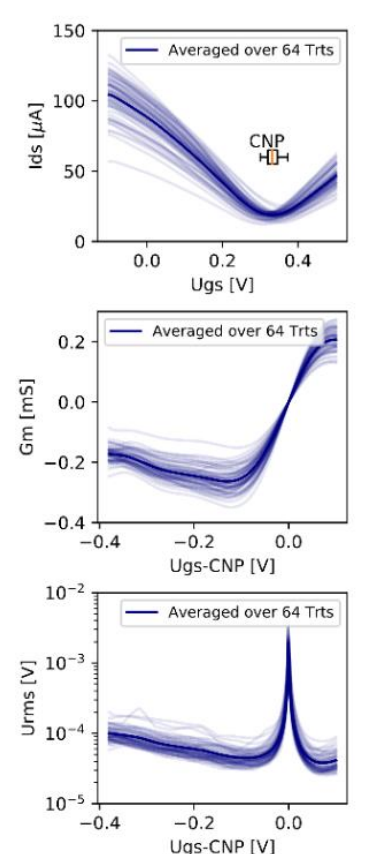

Ids $[\mu \mathrm{A}]$

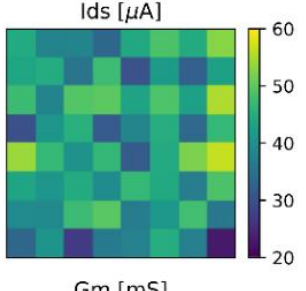

$\mathrm{Gm}[\mathrm{mS}]$

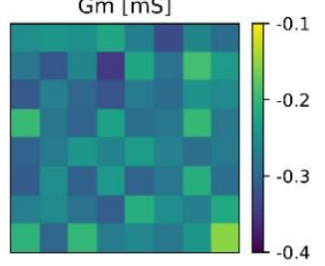

Urms [V]

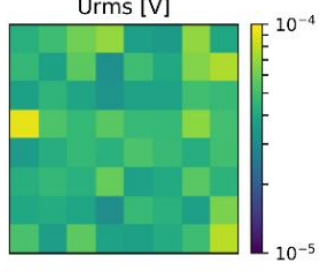

C

e
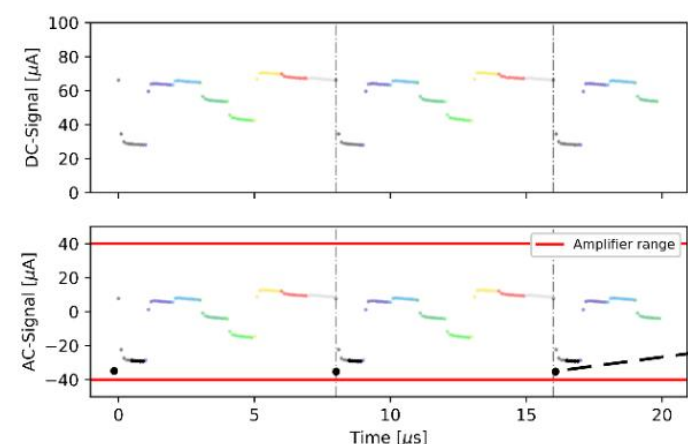

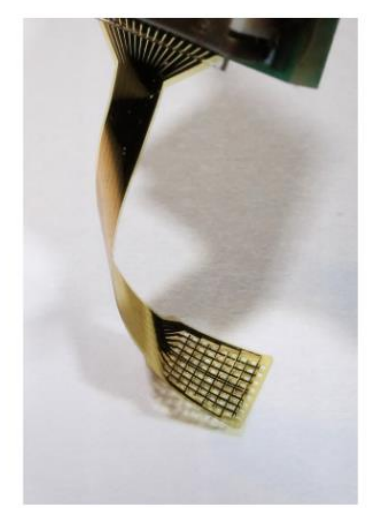

d DAQ-Card

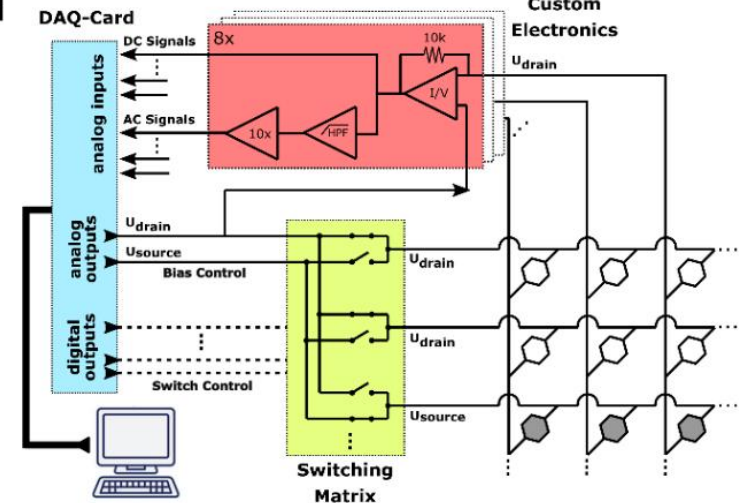

f 0.5 - Reconstructed signal for single gSGFET

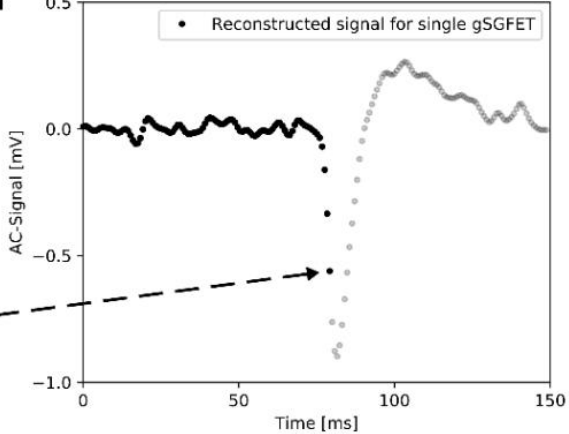

Figure 1. Multiplexed gSGFET $\boldsymbol{\mu E C o G - a r r a y : ~ a . ~ L a y o u t ~ a n d ~ c r o s s ~ s e c t i o n ~ o f ~ f l e x i b l e ~ E C o G ~ a r r a y ~ w i t h ~} 64$ gSGFETs showing the grid construction with two metal layer and tapered via-holes (Figure S1 in supporting information) crossing the PI interlayer. b. gSGFET's transfer curve showing the change of drain-source current with applied gate bias ( $\mathrm{I}_{\mathrm{ds}}-\mathrm{U}_{\mathrm{gs}}$, measured at $\left.\mathrm{U}_{\mathrm{ds}}=100 \mathrm{mV}\right)$, its transconductance $\left(\mathrm{G}_{\mathrm{m}}-\mathrm{U}_{\mathrm{gs}}\right)$ and the device's equivalent gate noise $\left(\mathrm{U}_{\mathrm{rms}}-\mathrm{V}_{\mathrm{gs}}\right)$. Also, the respective distribution of each parameter at peak $\mathrm{G}_{\mathrm{m}}$ (bias point yielding highest absolute transconductance) across the array is being shown in the adjacent color map. c. Picture of flexible probe after release from Si support wafer. d. Schematic of discrete multiplexing setup using a DAQ-card for bias control and read-out (blue) and a custom-build PCB board for switching (yellow), filtering and current-to-voltage conversion (red). e. Acquired DC and AC raw signal for one column showing eight distinct current levels corresponding to the transistors in each row, which repeat for every cycle (grey dashed line). Due to the high gain (100k) applied to the AC component of the signal, the dynamic amplifier range sets a limit to the allowable current mismatch between devices, before amplifier saturation occurs (solid red line). f. From the 10 data points taken for each transistor per cycle, an average value of the last 5 points is taken, to yield one sample in the final reconstructed signal for each sensor. 
Figure $1 \mathrm{~b}$ shows a performance and homogeneity assessment of the gSGFET array. The transfer curve, namely the change of drain-source current $I_{d s}$ with applied gate bias $U_{g s}$ is presented as an averaged value and for all 64 individual devices, with the boxplot indicating the variation of charge neutrality point (CNP). Also, the transconductance $\mathrm{Gm}$ is shown (normalized to each transistor's CNP), which is defined as the first derivative of $\mathrm{I}_{\mathrm{ds}}$ over $\mathrm{U}_{\mathrm{gs}}$. Of crucial importance to any sort of sensor array is the signal-to-noise ratio (SNR) and its homogeneity across recording sites. The SNR in the gSGFET is mainly affected by two physical parameters: the transconductance, which sets the transistor's sensitivity to signal fluctuations at the gate and the intrinsic device noise integrated over the relevant frequency range $\mathrm{I}_{\mathrm{rms}}$. A common figure of merit to quantify the gSGFET's SNR is the $U_{\text {rms }}$ value $\left(I_{r m s} / G_{m}\right)$, which represents the equivalent gate noise below which signals are undetectable. As can be seen, the $U_{r m s}$ values of the gSGFETs on the array, are around $40 \mu \mathrm{V}_{\text {rms }}$ (for the frequency band from $1 \mathrm{~Hz}$ to $100 \mathrm{~Hz}$ ), which allows the detection of local field potentials (typical amplitude above $100 \mu \mathrm{V})$ from the surface of the brain. The distribution across the array of each parameter $\left(I_{d s}, G_{m}, U_{r m s}\right)$, calculated at the bias point of highest absolute transconductance (peak- $G_{m}$ ), where the device shows lowest $\mathrm{U}_{\mathrm{rms}}$, is shown in the corresponding color maps. With yields above $90 \%$ on a 4 -inch wafer (each wafer carrying 14 probes) and large, evenly distributed SNR across all recording sites, the gSGFET technology shows high maturity and homogeneity, which is a key requirement to enable multiplexed addressing schemes.

The most common type of multiplexing is time-division multiplexing (TDM), which refers to a technique of sequentially addressing the columns of a sensor array, while continuously measuring from its rows. Such addressing is normally being achieved by switches, which can be placed either directly on the array or externally.[14, 17, 18] While placing the switches directly on the array has the advantage of reducing inter-site crosstalk it requires complex fabrication of flexible switching technology, as previously mentioned.[17] Complementing arrays of passive sensing elements (i.e. electrodes) with an external addressing configuration is challenging, as their recorded voltage signal is prone to pick up noise; if the preamplifier is placed far from the electrode, additional buffer transistors are required to convert the signal into current and feed it to the read-out circuitry. The use of active sensing elements (i.e. transistors) offers a great benefit here, as they feature an intrinsic voltage-to-current conversion, making the recorded signal more robust to noise. A schematic representation of the addressing and acquisition methodology is shown in Figure 1d. For this purpose, a custom-build PCB board consisting of two main functional blocks has been developed using discrete electronics. One functional block performs the filtering and amplification of the simultaneously acquired AC and DC signals; the other block selects the row to which the bias difference is applied to, using the external switching matrix for addressing. Figure 1e illustrates the acquired $\mathrm{AC}$ and $\mathrm{DC}$ raw input signals for a column of eight gSGFETs and how they can subsequently be used to reconstruct the original signal at each site (Figure 1f). Resistance mismatch between different transistors leads to current jumps when switching between transistors, which results in the different DC levels in the acquired signal (each gSGFET corresponds to one color in Figure 1e). The vertical dashed grey line in Figure 1e indicates the point at which all eight rows were addressed and a new readout cycle begins. At each site a total of 10 points is taken at $100 \mathrm{kHz}$ sampling speed; we discard the first 5 to avoid any switching artefacts and average the rest to a single data point resulting in $1.25 \mathrm{kHz}$ effective sampling rate. It should be here emphasized that array homogeneity carries an additional importance in multiplexed operation owing to two reasons. First, the tradeoff between applicable AC signal gain and transistor resistance mismatch; as previously mentioned, current jumps can exceed the dynamic range of the amplifier (indicated in Figure 1e) leading to saturation and signal loss. Second, the switching artefacts scale with the level of mismatch between devices, requiring longer stabilization times before reliable data points can be collected.

\subsection{Scalability and in-vitro assessment}

With the goal of creating a high-density, large-scale sensor array, an important discussion to undertake is the one on scalability of this technology. The most important aspects to consider here are the transient response of the transistor, which limits the achievable switching speeds, and the increase of inter-site crosstalk with array size and track resistance. To obtain distortion-free, high-quality recordings in multiplexed operation, it is important to allow sufficient stabilization time after switching, for the transistor to adjust to the changed drain-source bias conditions. This stabilization time is device specific and generally scales with channel length and carrier mobility. Owing to the high carrier mobility of graphene, which is for our devices $1000-2000 \mathrm{~cm}^{2} \mathrm{~V}^{-1} \mathrm{~s}^{-1}$ but can potentially reach up to $350000 \mathrm{~cm}^{2} \mathrm{~V}^{-1} \mathrm{~s}^{-1}$, the transient response of the gSGFET is extremely short (Figure 2a) compared to other technologies (e.g. transistors based on silicon or conducting polymers).[23] The ON-state is reached before $1 \mu \mathrm{s}$, which is the time resolution of the used measurement equipment (NI DAQ-Card X-Series 6363); for a channel length of $50 \mu \mathrm{m}$ and a carrier mobility of $2000 \mathrm{~cm}^{2} \mathrm{~V}^{-}$ ${ }^{1} \mathrm{~s}^{-1}$ the actual stabilization time from a time-of-flight 
estimation is expected to be in the range of hundred nanoseconds. Such fast device stabilization can eventually allow switching speeds beyond $1 \mathrm{MHz}$ (our current configuration is limited by the electronics to $10 \mathrm{kHz}$ ). Thus, much larger arrays could be operated this way while maintaining enough sampling speed $(>10 \mathrm{kHz})$ to record even high frequency activity such as spikes. Due to the solutiongated nature of the device, a small drift occurs during the $\mathrm{ON}$ state of the device which is tentatively associated to the migration of ions when the bias conditions in the channel change under switching. This phenomenon happens at a much slower time scale due to the low ionic mobility; however, it does only create a minor inaccuracy in the measured DC current and does not impact the AC recordings.

As previously stated, the use of external switches bears the disadvantage of not allowing to cut the connections to nonaddressed sensors within the array, which leads to the socalled crosstalk. The lack of on-site switches opens alternative current pathways leading to current contribution from adjacent sites which carry signals from the respective gates and are wrongly attributed to the measured sensor (see Figure $2 b$ ). Such crosstalk can be strongly reduced and, in the ideal case of vanishing track resistance, even fully suppressed by setting the bias point of the non-addressed rows to the same voltage as the data lines, thus preventing any current flow through non-addressed lines.[18] However, in real-world applications the issue of finite track resistance cannot be completely avoided, as the requirements of high sensor density and probe flexibility limit the allowed width and thickness of the metal lines. The presence of these additional resistances create local potential drops, driving current through otherwise suppressed pathways whose amplitude depends on the ratio between track resistance $\left(\mathrm{R}_{\text {track }}\right)$ and transistor resistance $\left(\mathrm{R}_{\mathrm{gSGFET}}\right)$ as well as the number $n$ of columns and rows in the array. To quantify crosstalk in the gSGFET array experimentally, lateral Aggates and confined pads of solid polyelectrolyte were placed at each gSGFET of a $2 \times 2$ array by means of inkjet printing (Figure 2c). This approach allows for selective gate control, which is not possible in a shared liquid electrolyte. Figure $2 d$ shows the measured signal at each of the transistors, with a test signal (sine wave of $10 \mathrm{~Hz}$ frequency and $30 \mathrm{mV}$ amplitude) only being applied to one of them (black circle in Figure 2c). Sensors on the same column (red curve in Figure 2d) or row (dark-red curve in Figure 2d) as the applied signal are the ones with the largest impact of crosstalk, showing a crosstalk level of $-40 \mathrm{~dB}$ at peak transconductance compared to the signal amplitude.

Diagonally placed sensors (orange curve in Figure 2d) are much less affected; however, in the latter case the exact level of crosstalk cannot be extracted with accuracy because the signal lies below the floor noise of the electronics. To validate this estimation of the crosstalk, we compared the experimental data with the results obtained from a PSpice simulation of a gSGFET array in which we used a standard p-type MOSFET element tailored to fit the gSGFET's transfer curve (Figure $\mathrm{S} 2 \mathrm{a}-\mathrm{b}$ in supporting information). Figure 2e shows the aggregated crosstalk on a single site depending on the track resistance and the array size, assuming identical signals on all remaining sites of the array which sum up to the total crosstalk value. The PSpice model validates the expected near-linear relation with both track resistance and array size. For a $2 \times 2$ array with $20 \Omega$ track resistance and a gSGFET resistance of $1,25 \mathrm{k} \Omega$, both the experiment and the simulation model yield a crosstalk of $-40 \mathrm{~dB}$. Extrapolating to an array of size $32 \times 32$ (1024 sensors), a crosstalk lower than $-20 \mathrm{~dB}$ can be obtained by reducing the track resistance below $5 \Omega$ or by increasing the resistance of the gSGFET. Such track resistance reduction could be achieved by increasing metal track thickness, using higher number of stacked metal layers and, most importantly, relaxing constraints on probe dimensions. While the ECoG array in this work was designed for application in rodents, which imposes strict size restrictions due to the dimensions of the craniotomy, many other application (e.g. neural probes for large animals or humans) would loosen those significantly. To validate the fidelity of the gSGFET recordings in the multiplexed operating mode, the recording quality must be compared to the one obtained in steady, non-multiplexed operation. For instance, the rapid switching between devices can potentially increase the noise or generate artefacts in the multiplexed operation mode. Figure $2 f$ compares recordings of an artificially generated electrocardiogram signal (ME-WSG, Multichannel Systems), containing components of different frequencies. The multiplexed and non-multiplexed representation of both test signals are nearly identical, suggesting that the rapid sequential addressing by the multiplexed mode does not generate any visible artefacts, neither in the low nor in the mid frequency band. The rootmean-square value of both recordings is also compared, showing equivalent SNR ratio (Figure $2 \mathrm{~g}$ ) in both acquisition modes. 
a
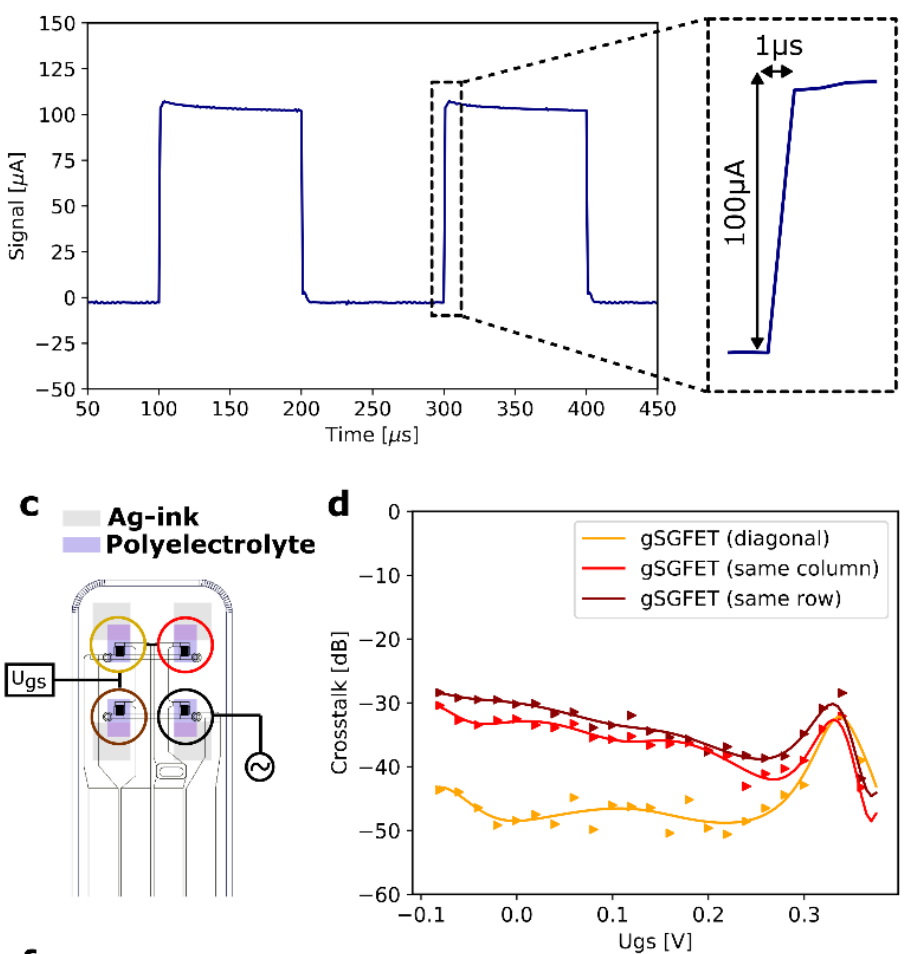

$\mathbf{f}$
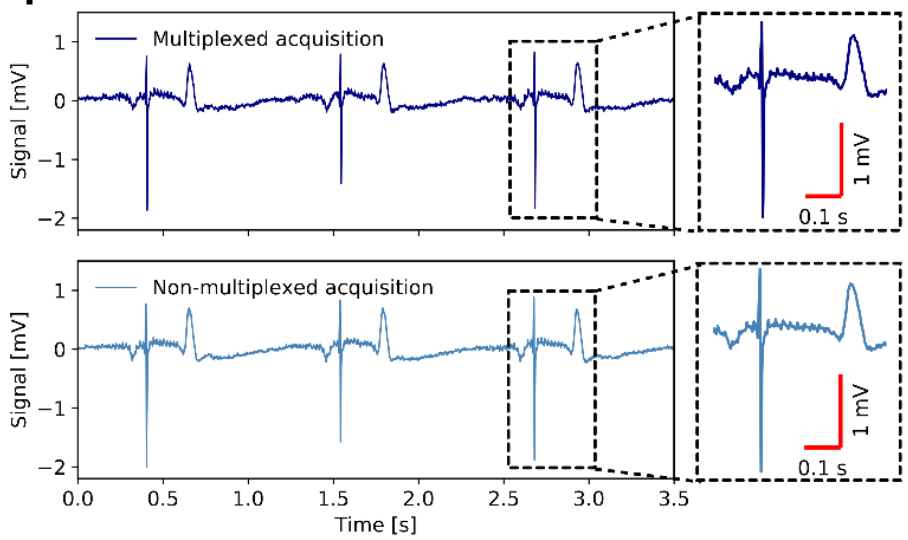

b
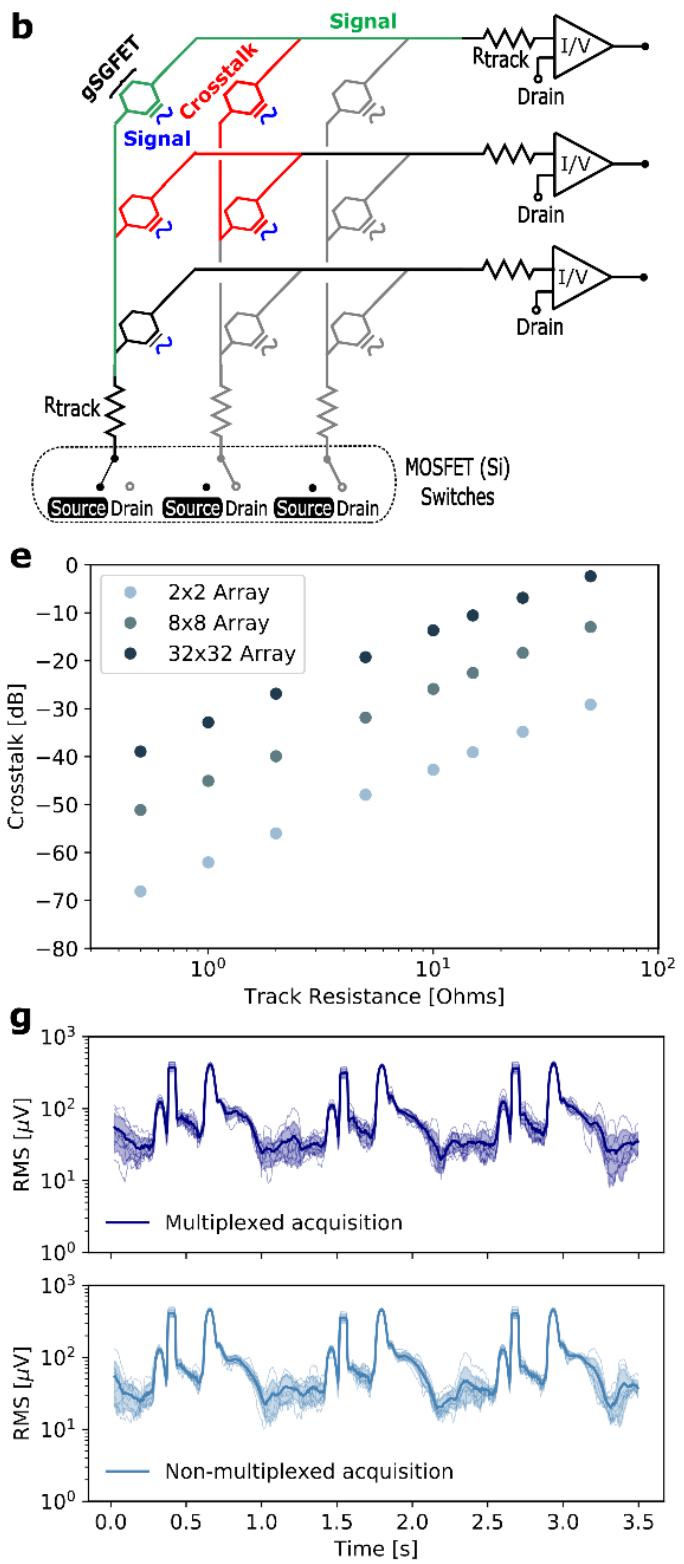

Figure 2. Scalability and in-vitro assessment: a. Transient response of gSGFET when switched between ON and OFF state, showing short stabilization time of the device. Response below $1 \mu$ s cannot be resolved due to sampling rate limitation of the setup (1MHz). b. Origin of crosstalk in array without on-site switches and finite track resistance. Biasing to $\mathrm{U}_{\text {drain }}$ of non-addressed columns significantly decreases the amount of crosstalk. c. Location of measured gSGFETs on a 2x2 probe for crosstalk evaluation, utilizing inkjet printing to pattern confined gates consisting of $\mathrm{Ag}$ and polyelectrolyte pads. d. Crosstalk versus gate bias when applying a test signal (sinewave $30 \mathrm{mV}, 10 \mathrm{~Hz}$ ) to a single gSGFET and measuring the signal on each of the adjacent sensors. The devices in the same column and row as the one, to which the test signal is applied to, show a crosstalk of $-40 \mathrm{~dB}$ while the device on the diagonal shows crosstalk lower than $-50 \mathrm{~dB}$ (below floor noise of electronics). e. Simulated (PSpice) crosstalk for a 2x2, $8 \times 8$ and 32x32 arrays in dependence of the track resistance. The aggregated crosstalk value is presented assuming a superposition of identical signals on all but the probed sensor. f. Recordings of one gSGFET on the same 8x8 $\mu \mathrm{ECoG}$-array, acquired in either multiplexed or steady (non-multiplexed) acquisition. An artificial electrocardiogram signal was used to compare the fidelity of both acquisition modes. In both cases, the same averaging method was applied (cycles of 10 points, discarding first 5 points and averaging last 5 points), to ensure comparability. g. For each acquisition mode, the root-mean-square (RMS) value is calculated as the mean value of all $8 \mathrm{gSGFETs}$ on one column, indicating the signal-to-noise ratio of the recorded signal. 


\subsection{In-vivo validation}

Having confirmed the recording fidelity of the gSGFET in the multiplexed operation compared to the standard nonmultiplexed acquisition mode, we demonstrate the unimpaired sensitivity of the multiplexed $\mu \mathrm{ECoG}$-array towards biological signals. Electrocortiography is primarily used to record local field potentials (LFPs) from the surface of the brain, which originate from spatio-temporal summation of transmembranecurrents generated by synaptic and active conductances, excluding the action potentials. $[29,30]$ The characteristic spectral content of the LFP signal reflects the time scale of network synchronization and ranges from $<1 \mathrm{~Hz}$ to hundreds of Hz. Figure 3a shows a time trace of spontaneous LFP activity in an anesthetized Long Evans rat, recorded in an acute in-vivo experiment using an array of gSGFETs operated in the multiplexed mode (more details in methods section). Under deep anaesthesia, such cortical LFP reflects slow oscillations that are highly coherent across the whole cortical mantle; indeed, no significant differences in signal shape can be seen across all the 64 sensors in the array.[31] To benchmark the recording capabilities of the gSGFET array against other state-of-the-art technologies, a NeuroNexus ECoG array (with 32 circular platinum electrodes of $100 \mu \mathrm{m}$ diameter) was simultaneously placed on the opposite hemisphere (Figure S3 in supporting information). Figure 3c$\mathrm{d}$ show recordings and corresponding spectrograms from an electrode and a gSGFET, displaying the expected synchronicity in activity of both hemispheres. Filtering the signals from $0.1 \mathrm{~Hz}$ to $200 \mathrm{~Hz}$ (dark-blue and red curve in Figure 3c), a clear difference in signal shape can be noticed. This difference reflects the high-pass filter of the AC-coupled headstage used for passive ECoG recordings (see Experimental Section), which is necessary to prevent amplifier saturation due to drifts; also the high impedance of passive ECoG electrodes at low frequency leads to reduced gain in such signals, which results in an attenuation of the infraslow frequency content in the LFP signal.[22] Removing these low-frequency components from the gSGFET signal (green curve in Figure 3c), it is possible to validate that both technologies show very similar recordings of the LFP activity as can also be seen in the corresponding spectrograms.

Visually evoked LFP activity exhibits a well-defined spatial topography and thus can be used for validating the mapping capabilities of the multiplexed gSGFET technology.[32] Figure $3 \mathrm{~b}$ shows the averaged response over 10 consecutive evoked events recorded with an array of gSGFETs in the multiplexed mode. Visually evoked activity typically exhibits a clear response with a delay of $40 \mathrm{~ms}$ after both the $\mathrm{ON}$ - and OFF-switching of the stimulus (Figure 3e). The recordings show a main peak (ON response) lasting until $70 \mathrm{~ms}$ after the stimulation with and peak amplitude of $500 \mu \mathrm{V}$. Hence, signal shape and amplitude are in nice agreement with previously reported results of non-multiplexed gSGFETs.[20] Further, Figure $3 \mathrm{f}$ presents the spatial distribution of both amplitude and time-delay of the ON-peak. The earliest response is detected on the lower end of the array which represents sensors directly placed on the primary visual cortex (V1) where the activity originates, and spreads then radially towards other higher visual cortical areas. However, highest peak-amplitude is measured in the centre-left region (secondary visual cortex, V2) which likely reflects a different magnitude and proximity current dipole, that gives rise to the surface LFP (LGN input to LIV in V1 vs V1 input to L2/3 pyramidal cells in V2).[33]

In addition, to its capability to record LFPs, the gSGFETs exhibit a unique sensitivity towards slow and infra-slow (below $0.1 \mathrm{~Hz}$ ) signals, which in the case of passive electrode recordings are hidden by baseline drifts and the impedancerelated loss of gain at low frequency.[22] In order to confirm that multiplexed acquisition preserves signal quality in this frequency band, we have investigated recordings of a cortical spreading depression (CSD). CSDs emerge due to a cellular depolarization of neurons and astrocytes which is associated to brain injury and migraines among others.[34] Here, the CSD was artificially triggered by injecting $\mathrm{KCl}$ into the cortex that caused a slowly propagating wave moving across the cortex. Figure $3 \mathrm{~g}$ depicts the recording of a gSGFET in the array. The DC component of the signal shows the characteristic large shift of $15 \mathrm{mV}$ amplitude. The AC component and its corresponding spectrogram (Figure $3 \mathrm{~h}$ ) reveals a silencing of the high-frequency activity during the event, caused by the cellular depolarization and which is characteristic for the CSD. The maps below (Figure 3i) show the respective position of the depolarising wave at different times after KCL injection, moving from the top right to the bottom left at about $7 \mathrm{~mm}$ per minute speed. 
a

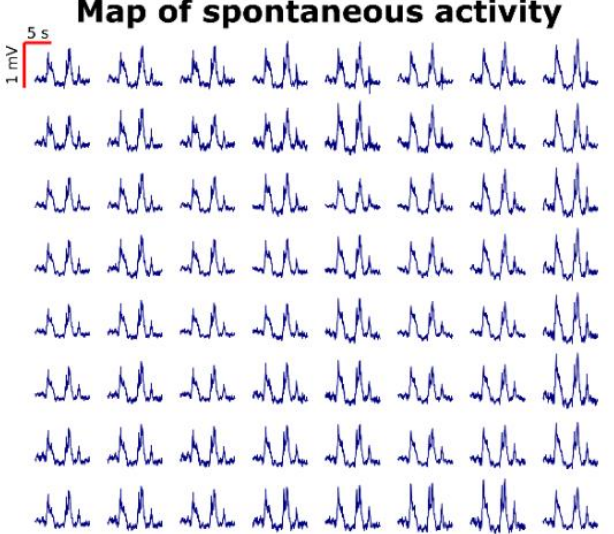

b ${ }_{100 \mathrm{~ms}}$ Map of visually evoked response

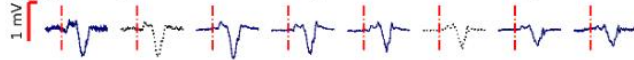

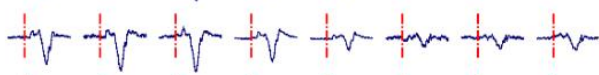

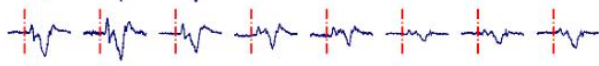

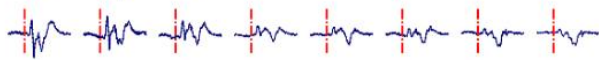

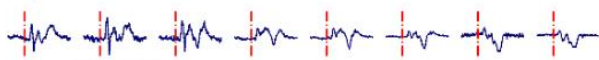

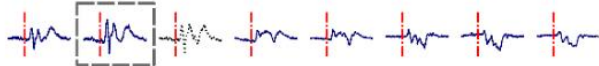

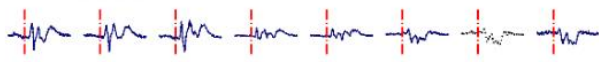

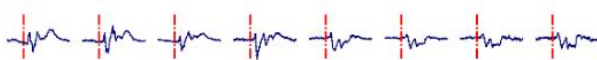

Benchmark against commerical ECoGs
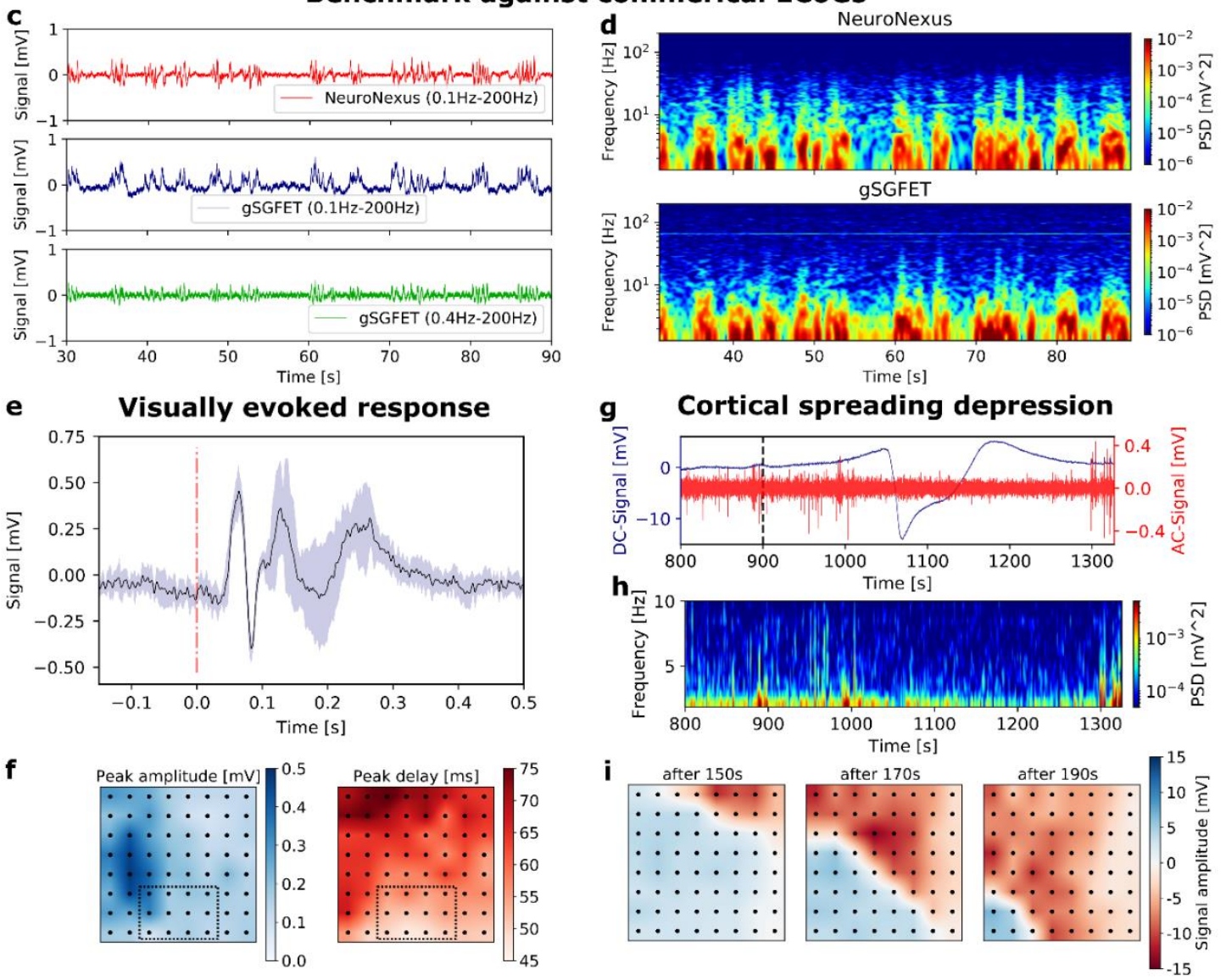

Figure 3. In-vivo validation of multiplexed gSGFET $\mu$ ECoG-array: a. Map of spontaneous LFP activity showing near identical shape across all sites. b. Map of visually evoked activity averaged over 10 consecutive events (dashed red line indicates timing of optical stimulus; dashed black curves are interpolated values from neighbouring sites for non-functioning sensors. c. Recording trace derived from AC-coupled passive ECoG-array (NeuroNexus, platinum electrode) as well as DC and AC-filtered gSGFET's. Note prominent infraslow oscillations (0.1$0.4 \mathrm{~Hz}$ ), which are not visible in the AC electrode recordings. d. Corresponding spectrograms of both, NeuroNexus and gSGFET recordings. e. Visually evoked response recorded on a single sensor (dashed grey box in Figure 3b), with an ON- and OFF-response of 50 ms delay and $500 \mu \mathrm{V}$ peak amplitude. f. Array maps displaying the distribution of amplitude and time-delay of the ON-peak response across all sites. While the shortest delay is measured for the sensors placed directly on the primary visual cortex (V1), the highest peak-amplitude is seen in the secondary visual cortex (V2). g. DC-signal (blue, lowpass-filtered below $20 \mathrm{~Hz}$ ) and AC signal (red, bandpass-filtered between $1-20 \mathrm{~Hz}$ ) of a single gSGFET during the cortical spreading depression (CSD). A strong DC shift of $-15 \mathrm{mV}$ occurs simultaneously with a silencing of highfrequency activity, which is characteristic for a CSD. h. Corresponding spectrogram of CSD event to validate the silencing. i. Array map showing the propagating front of the spreading depolarization wave across the array from the right upper to the left lower corner. 


\section{Conclusion}

This work demonstrates the compatibility of graphene solution-gated field-effect transistors (gSGFETs) with timedivision multiplexed acquisition mode, utilizing a strongly simplified addressing concept to overcome the hurdle of excessively complex device fabrication. The superiority of graphene over silicon and organic polymers for high-speed applications makes the gSGFET an ideal device for multiplexed sensor arrays. In-vitro and in-vivo assessments confirm the fidelity of broad-band signal representation (infraslow oscillations and local field potentials) in multiplexed operation, with signal quality comparable to the state-of-theart of commercially available neuro-sensors. Consequently, next to their potential as efficient transducers of neural activity, gSGFETs show great promise as a building block for multiplexed brain-machine interfaces of high sensor count. Thus, this work represents an important cornerstone in the development of large-scale, flexible gSGFET $\mu$ ECOG arrays capable of providing high resolution mappings of neural activity to control neuroprosthetics and to help exploring the operation and functionalities of the brain.

\section{Acknowledgements}

This work has received funding from the European Union's Horizon 2020 research and innovation programme under grant agreements No 785219 (Graphene Flagship Core Project 2) and $\mathrm{N}^{\circ} 732032$ (BrainCom). We also acknowledge funding form Generalitat de Catalunya 2017 SGR 1426, and the 2DTecBio (FIS2017-85787-R) funded by Ministry of Science, Innovation and Universities, the State Research Agency (AEI) and the European Regional Development Fund (FEDER/UE). The ICN2 is supported by the Severo Ochoa Centres of Excellence programme, funded by the Spanish Research Agency (AEI, grant no. SEV-2017-0706), and is funded by the CERCA programme / Generalitat de Catalunya. R.G.C. is supported by the International $\mathrm{PhD}$ Programme $\mathrm{La}$ Caixa - Severo Ochoa (Programa Internacional de Becas "la Caixa"-Severo Ochoa). This work has made use of the Spanish ICTS Network MICRONANOFABS partially supported by MICINN and the ICTS 'NANBIOSIS', more specifically by the Micro-NanoTechnology Unit of the CIBER in Bioengineering, Biomaterials and Nanomedicine (CIBERBBN) at the IMB-CNM. GG and SS thank financial support from the MINECO/FEDER CTQ2015-65439-R project. SS is supported by FEDER funds managed by the Catalan Secretary of Universities and Research through project PROD-0000114 (Enterprise and Knowledge, Industry Department, Generalitat de Catalunya).

\section{References}

1. Lee B, Kramer D, Salas MA, Kellis S, Brown D, Dobreva T, Klaes C, Heck C, Liu C, Andersen RA (2018) Engineering artificial somatosensation through cortical stimulation in humans. Front Syst Neurosci 12:1-11

2. Ajiboye $A B$, Willett FR, Young DR, et al (2017) Restoration of reaching and grasping movements through brain-controlled muscle stimulation in a person with tetraplegia: a proof-of-concept demonstration. Lancet 389:1821-1830

3. Moxon KA, Foffani G (2015) Brain-machine interfaces beyond neuroprosthetics. Neuron 86:55-67

4. Orsborn AL, Pesaran B (2017) Parsing learning in networks using brain-machine interfaces. Curr Opin Neurobiol 46:76-83

5. Clancy KB, Koralek AC, Costa RM, Feldman DE, Carmena JM (2014) Volitional modulation of optically recorded calcium signals during neuroprosthetic learning. Nat Neurosci 17:807809

6. Shanechi MM (2019) Brain-machine interfaces from motor to mood. Nat Neurosci 22:15541564

7. Mayberg HS, Lozano AM, Voon V, Mcneely HE, Seminowicz D, Hamani C, Schwalb JM, Kennedy SH (2005) Deep Brain Stimulation for Treatment-Resistant Depression ing electrical stimulation of the subgenual cingulate. Neuron 45:651-660

8. Holtzheimer PE, Husain MM, Lisanby $\mathrm{SH}$, et al (2017) Subcallosal cingulate deep brain stimulation for treatment-resistant depression: a multisite, randomised, sham-controlled trial. The Lancet Psychiatry 4:839-849

9. Dagamseh AMK, Wiegerink RJ, Lammerink TSJ, Krijnen GJM (2012) Towards a high-resolution flow camera using artificial hair sensor arrays for flow pattern observations. Bioinspiration and Biomimetics. https://doi.org/10.1088/17483182/7/4/046009 
10. Anumanchipalli GK, Chartier J, Chang EF (2019) Speech synthesis from neural decoding of spoken sentences. Nature 568:493-498

11. Raducanu BC, Yazicioglu RF, Lopez CM, et al (2016) Time multiplexed active neural probe with 678 parallel recording sites. Eur Solid-State Device Res Conf 2016-Octob:385-388

12. Jun JJ, Steinmetz NA, Siegle JH, et al (2017) Fully integrated silicon probes for high-density recording of neural activity. Nature 551:232236

13. Angotzi GN, Boi F, Lecomte A, Miele E, Malerba M, Zucca S, Casile A, Berdondini L (2019) SiNAPS: An implantable active pixel sensor CMOS-probe for simultaneous large-scale neural recordings. Biosens Bioelectron 126:355-364

14. Eversmann $B$, Jenkner $M$, Hofmann $F$, et al (2003) A $128 \times 128$ CMOS Biosensor Array for Extracellular\rRecording of Neural Activity. leee J Solid-State Circuits, 38:2306-2317

15. Khodagholy D, Gelinas JN, Thesen T, Doyle W, Devinsky O, Malliaras GG, Buzsáki G (2015) NeuroGrid: Recording action potentials from the surface of the brain. Nat Neurosci.

https://doi.org/10.1038/nn.3905

16. Escabi MA, Read HL, Viventi J, et al (2014) A high-density, high-channel count, multiplexed ECoG array for auditory-cortex recordings. J Neurophysiol 112:1566-1583

17. Viventi J, Kim DH, Vigeland L, et al (2011) Flexible, foldable, actively multiplexed, highdensity electrode array for mapping brain activity in vivo. Nat Neurosci 14:1599-1605

18. Lee W, Kim D, Matsuhisa N, Nagase M, Sekino M, Malliaras GG, Yokota T, Someya T (2017)

Transparent, conformable, active multielectrode array using organic electrochemical transistors. Proc Natl Acad Sci 201703886

19. Lee W, Kim D, Rivnay J, Matsuhisa N, Lonjaret $T$, Yokota T, Yawo H, Sekino M, Malliaras GG, Someya T (2016) Integration of Organic Electrochemical and Field-Effect Transistors for
Ultraflexible, High Temporal Resolution Electrophysiology Arrays. Adv Mater 28:97229728

20. Hébert C, Masvidal-Codina E, Suarez-Perez A, et al (2018) Flexible Graphene Solution-Gated Field-Effect Transistors: Efficient Transducers for Micro-Electrocorticography. Adv Funct Mater 28:1-15

21. Kostarelos K, Vincent M, Hebert C, Garrido JA (2017) Graphene in the Design and Engineering of Next-Generation Neural Interfaces. Adv Mater 29:1-7

22. Masvidal-Codina E, Illa X, Dasilva M, et al (2019) High-resolution mapping of infraslow cortical brain activity enabled by graphene microtransistors. Nat Mater 18:280-288

23. Banszerus L, Schmitz M, Engels S, Dauber J, Oellers $\mathrm{M}$, Haupt F, Watanabe $\mathrm{K}$, Taniguchi T, Beschoten B, Stampfer C (2015) Ultrahighmobility graphene devices from chemical vapor deposition on reusable copper. Sci Adv 1:1-7

24. Stavrinidou $E$, Leleux $\mathrm{P}$, Rajaona $\mathrm{H}$, Khodagholy $D$, Rivnay J, Lindau M, Sanaur S, Malliaras GG (2013) Direct measurement of ion mobility in a conducting polymer. Adv Mater 25:4488-4493

25. Dankerl M, Hauf M V., Lippert A, et al (2010) Graphene solution-gated field-effect transistor array for sensing applications. Adv Funct Mater 20:3117-3124

26. Li X, Cai W, An J, et al (2009) Large-area synthesis of high-quality and uniform graphene films on copper foils. Science (80- ) 324:13121314

27. Hess LH, Hauf M V., Seifert M, Speck F, Seyller T, Stutzmann M, Sharp ID, Garrido JA (2011) High-transconductance graphene solutiongated field effect transistors. Appl Phys Lett 99:2009-2012

28. Hess LH, Jansen M, Maybeck V, Hauf M V., Seifert M, Stutzmann M, Sharp ID, Offenhäusser A, Garrido JA (2011) Graphene transistor arrays for recording action potentials from electrogenic cells. Adv Mater 23:50455049 
29. Buzsáki G, Anastassiou CA, Koch C (2012) The origin of extracellular fields and currents-EEG, ECoG, LFP and spikes. Nat Rev Neurosci

13:407-420

30. Pesaran B, Vinck M, Einevoll GT, Sirota A, Fries

$P$, Siegel $M$, Truccolo $W$, Schroeder CE, Srinivasan R (2018) Investigating large-scale brain dynamics using field potential recordings: Analysis and interpretation. Nat Neurosci 21:903-919

31. Sirota A, Buzsáki G (2005) Interaction between neocortical and hippocampal networks via slow oscillations. Thalamus Relat Syst 3:245-259

32. Katzner S, Nauhaus I, Benucci A, Bonin V, Ringach DL, Carandini M (2009) Local Origin of Field Potentials in Visual Cortex. Neuron 61:3541

33. Michalareas $G$, Vezoli J, van Pelt S, Schoffelen JM, Kennedy H, Fries P (2016) Alpha-Beta and Gamma Rhythms Subserve Feedback and Feedforward Influences among Human Visual Cortical Areas. Neuron 89:384-397

34. Cozzolino O, Marchese M, Trovato F, Pracucci E, Ratto GM, Buzzi MG, Sicca F, Santorelli FM (2018) Understanding spreading depression from headache to sudden unexpected death. Front Neurol 9:1-13 